\title{
The Ethos of Artificial Intelligence as a Legal Personality in a Globalized Space: Examining the Overhaul of the Post-liberal Technological Order
}

\author{
Abhivardhan $^{(\bowtie)}(\mathbb{1}$ \\ Indian Society of Artificial Intelligence and Law, 8/12, Patrika Marg, Civil Lines, \\ Allahabad 211001, India \\ abhivardhan@isail.in
}

\begin{abstract}
The categorical ethos of artificial intelligence is influenced by its basic structure, which defines its due purpose as a legal personality, challenging the conventional standards of law and justice in a globalized world. Recent developments show a precedential growth in the need-perspective of the AI industry, thereby influencing governance and corporate operations and their legal side in cross-cultural avenues. The determinant outlining of artificial intelligence as a legal personality rests on its probabilistic nature, which yet can be limited to the jurisprudential scope of AI-based on the ethos of the utilitarian approach involving the anthropocentric innovations for artificial intelligence. The dynamic nature of AI, however, in the proposition, is capable of a full-fledged and anthropomorphic legal representation and interpretation, which is hard to find in D9 and certain developing countries, which poses special risks to the generic legal infrastructure of a democratic polity to understand the dynamic and self-transformative nature of artificial intelligence in the age of globalization.

The paper is thus based on the proposition that the ethos involving the legal infrastructure and persona of artificial intelligence is traceable and easier in deterministic mechanisms by regarding and extending stable \& constitutive approaches to dissect the legal challenges connected with the redemptions implicated with the lack of a full-fledged regard and scope of the legal personality of AI. The approaches in due proposition are (a) anthropomorphisation; (b) naturalization; (c) techno-socialization; and (d) enculturation. Further, the paper analyses on the challenges to determine the problematic implications awaited by the influence of populism, protectionism, data-centred digital colonialism and technology distancing and proposes suggestions based on the four approaches to counter the minimal effects of the implications. The conclusions of the paper rest on the argument that in the case of a post-liberal order, the ethos of AI can be protected and diversified by adapting with the appreciation of the ethos of globalization, giving adequate, constitutive and reasonable space to the identity-led implications of national identity \& diluting the monopolistic influence of the utilitarian approach to artificial intelligence.
\end{abstract}

Keywords: International relations - Law and technology ethics - AI ethics · Behavioural economics · Identity ethics · Consumerism 


\section{Introduction}

The economics of development in a globalized world using technology for imparting the cause of a welfare state is connected with the usefulness of technocratic governance among nation-states in order to convert the stature of technology from being a mere tool or need to become an integral asset of human lives and the constructive systems of governance among public and private actors. Developed countries, therefore, encourage technocratic governance sponsoring transparency, accountability and technical cum humanized regulation \& regularization of 'technological assets'.

Artificial Intelligence is gradually becoming a relevant technology asset for knowledge-driven societies and governments \& even in cases where knowledge-driven policies [1] and methodologies are yet to be transformed. Nevertheless, the legal transformation of AI is not limited to the planar legality defined in the constitutional and political systems across nation-states, and the barriers and infrastructures of conventional and proceduralist morality in these systems are plausibly hampered by a systemic, yet unparallel conceivable evolution of technology [2, 3]. The rules-based international order maintained and nurtured among nation-states is also suffering from various redemptions that are constitutional, legal, political and social by nature and practice. Generally, the international rule-based order is at the brink of balance between natural morality and state interests [4]. There are, however, existent certain legal, social and political redemptions that damage the inherent plausibility of technocratic governance and the commitment of a liberal order [5]. Thus, the advancement of technological assets is set out to face a postliberal globalized international order with the complications of redemptive decisions, modalities and foresight. Therefore, the groundwork for making technology integral to societies and lives is set to be affected by diplomatic and technological actors involved.

In case of AI, it is proposed in this paper that there exist three non-exhaustive and integral challenges to both corporate and state actors in developing and developed economies that shape the legal and socio-political ethos of the AI in a post-liberal order. The paper further analyses and proposes the essential aspects of the three challenges and proposes in its further sections four constitutive approaches for an ethical assessment of AI in law and policy-making measures, taking into consideration the rivalled nature of problems due to $\mathrm{AI}$ in developed and developing economies with conclusions.

\section{Challenges to Harmonization of AI}

The harmonization of technology as an asset to public welfare and rule of law is a predominant notion accepted among nation-states and the same. Various declarations, reports and policy documents focus on a harmonious AI, and they endorse a regularized and ethical usage and control of AI as a utility. There is literature available, including case studies, reports and opinions by experts and think-tanks, which show certain reflective aspects as to how should AI be harmonized for public welfare. These are certainly important aspects of a harmonized AI that is duly expected:

- AI must be robust, and bear fairness and accountability [6, 7];

- AI must respect privacy concerns by design and default $[6,8]$; 
- AI must be regulated and should be human-centric $[9,10]$;

- AI must respect the principles of legitimacy and constitutionalism [10, 11];

- AI must adhere to international human rights obligations [6];

- AI must reflect human-centric and individualistic interests;

- AI must be objective in its probabilistic operations [7];

The essential aspects of an ethical AI are not exhaustive and cannot be preconditioned, because the dynamic behaviour of AI cannot be confined by limited legal interpretation nor the natural aspect of scientific development can be averted. However, the problems in fulfilling the goals to achieve an ethical AI are not limited to the technological domain of AI, which involves technological ethics but have certain socio-political and legal implications as well. These problems emerge as real challenges to the issue and as per the propositions in the paper - are divided into three essential (non-exhaustive) cases, which are enumerated as follows:

1. the uncertain characteristics of populist politics in foreign and domestic policies;

2. the resistant measures are undertaken by governments under the policy and practice of legal, social and technological protectionism;

3. a data-truistic and data-centered digital colonialism of technology in economic and digital competence;

The further sub-sections lay down the social, political and legal challenges towards fostering an impeccable development of the AI ecosystem with suggestions provided.

\subsection{Effect of Populism on the Social and Technological Domains}

Populism is a political phenomenon, which involves the conception of popular sovereignty and the politics of redemption, which is not limited to democratic establishments, and some of its tactical practices can be seen in China, Russia and even Iran. Nevertheless, with the intent of dissecting ideological intent and welfare interests merged and represented by the leaders who subscribe or have gained power due to populism in politics, the propositions focus on the following reflections of populism in terms of due involvement and inspiration to the connoted domains of technology and society, taking into consideration the market economy perspective behind the development of AI as a technical utility and its ethical role:

- Populism involves a technical disruption of political and democratic confidence among law and policymakers, people and even at the integral levels of governmental agencies $[12,13]$;

- Populism in many countries involve opaque data protection and privacy ethics policymaking, demanding a closed implementation of data protection policies beyond ideological and political maneuvers of the policy-makers and relevant stakeholders due to lack of a clear, well-informed and well-concerted knowledge among the people who legitimize their leaders' political actions [14], whether by legal or illegal direct or indirect means; 
- Populism often in many countries involves the politics of redemption, which means that a chain of legal reforms or miscellaneous changes disrupt the policy status quos of a state;

- In many instances, the precedents of technological ethics and humanism assumed by the incumbent governments to foster consensual measures among the members of the international community are alleged to be or in reality, either reversed or potentially damaged [15];

Though these propositions are non-exhaustive, they are yet reflective of the challenges that law and policymakers, political and civic groups and people face these days.

\subsection{Influence of Legal, Economic and Technical Protectionism}

In issues related to politics, legal reforms and economics, it is essential that an undone or wrongly achieved act or exercisable policy must be reconvened and resolved adequately. Reasonableness is, therefore, an essential criterion that develops and legitimizes redemptive activities. In the case of AI, there are relative problems with rising implications evolved due to the principled practices of globalization and the conception of the market economy $[16,17]$.

While the role of globalization is centered to global capitalism, it respects and is centered at the idea of neoliberal economics. Other than economics, globalization endorses multilateral organizations over strategic partnerships and integrable legal measures, based on consensus and peacebuilding. However, there is a shift in the global consensus from a globalist approach to a protectionist approach when it comes to technological democratization. The proposed reflections of the current trends of protectionism in tech-centric diplomacy are enumerated as follows:

- Precedential to various redemptions, law and policymakers feel that the policies under globalization adopted by their state involving the regimes of data protection, accountability and trust are acting to fail because various nation-centric issues with respect to technology are being ignored;

- Corruptible and incredulous practices among globalized economies in governmental and non-state actors weaken the position of the stakeholders involved in tech-centric diplomacy to foster developmental approaches via technology;

- Lack of target-centric tech measures involves adverse implications in welfare activities. Tech measures are therefore central to entrepreneurial ethics and its design because they decide for a set of generations their education, skill development and employment opportunities as well as the policy that shapes the same.

- The market economy approach defeats indigenous interests, lacks replenishment in entrepreneurial ethics and design in developed and developing economies because it lacks a vision towards policing newer and improvable employment opportunities [17];

\subsection{Scope of Data-Centered and Data-Truistic Digital Colonialism}

Digital colonialism is a phenomenon that involves the supremacy of state and non-state actors in certain areas via domination in the digital activities of technocratic cyberspace. 
In the coming years, the phase of creation in the life of internet sustained changes and would continue with the phase of surveillance. The libertarian approach of internet governance is being modified by the technocratic approach of internet governance, making surveillance essential. Digital colonialism became inevitably a problem due to such implications of internet governance. While the role of protectionism and populism is essential to digital colonialism, yet the practicable aspects of digital colonialism have traversed vast, which may be hazardous. The proposed reflections of the current trends of digital colonialism in tech-centric diplomacy are enumerated as follows:

- Trust is an important currency in dealing with tech-centric diplomacy and innovation among the stakeholders involved in the process [16]. If issues of consensual importance related to the data protection regimes are not adequately settled, then it would certainly affect the moral capital of disruptive technologies;

- Technology distancing is a key tool to benefit digital colonialism [18], politically, socially and institutionally. The problem of digital colonization persists not with the libertarian approach of free speech and other relevant and connected liberties, and neither is the approach of technological and political liberalization is flawed. The persistent catalyst that drives the libertarian approach governance in globalization is conventional morality [5]. However, a lack of trust and autonomy among the individuals, who either control or become the data subjects endorses technology distancing, whereby empowering digital colonialism by gradual means;

It is proposed that there are four non-exhaustive approaches to assess and estimate the ethos involving the legal infrastructure and persona of artificial intelligence to dissect the legal and ethical challenges towards harmonizing AI with its aesthetic perspective and pragmatism.

\subsection{Hypothesis}

The hypothesis of the paper is based on the following corollaries:

- That the aesthetic component of AI can be estimated by four constitutive approaches to render an objective and entity-centric assessment of the AI as a human artefact;

- The four constitutive approaches to assess AI are (a) Anthropomorphisation, (b) Naturalization, (c) Techno-socialization, and (d) Enculturation;

- Anthropomorphisation, as proposed is the approach of accepting the substantive and operative attributes of AI, entailing a proper distinguishability of the attributes and adapting the operative attributes of AI in conformation with the transformable humanrelated attributes acquired through data subjects by constructive and stable estimation of the data processed;

- Naturalization, as proposed, is the approach of revising and improving the substantive attributes of AI-based on the stimulated data received towards a coherent, constitutive and peaceful adaptability of such transformable human-oriented attributes perceived from the data subject;

- Techno-socialization, as proposed, is the approach of making the environment for AI socialized, regularized and improved with better and constitutive processing of the data and the ethical channeling of the stimuli on which the AI is reliant; 
- Enculturation, as proposed, involves the approach of encouraging and harmonizing the identity-oriented footprints generated, learnt and manifestly determined by AI by its probabilistic mechanisms;

With the concept of the four approaches, the ethos of AI as a legal personality is proposed to be harmonized and constructive to ensure better, coherent and unbiased legal recognition and interpretation of the role, persona and activities of artificial intelligence. The additional propositions in the hypothesis are provided as follows:

- The recognition of $\mathrm{AI}$ as a juristic entity is required to be extended from being an industrial and economic unit to an organic technology that can possess certain limited yet applicable rights in conjugation with the human stimulus with which it is entitled to exist and remain to ensure its nature to be human-centric;

- The constitutive approaches are based on the argument that the dynamic yet opaque behaviour of AI must be decluttered with better and empathy-oriented measures, where the aesthetic ecosystem between AI and human entities are assessed carefully;

- Further, the receptive rights of the AI, (which are based partly on the technical and vicarious responsibilities of the AI \& partly on the entrepreneurial ethics and design as an inherently absolute and fundamental responsibility of the state and non-state actors, who are involved with that AI at any possible and foreseeable levels of human agency) \& the obscure and delicate privacies pre-emptively reserved by data subjects (human entities) must be reconciled;

- The natural and civil liberties of AI that are reservedly developed and exercised by the AI (whether recognized or not recognized by law), must be carefully adjudicated and must be properly bridged with the privacy rights of human entities as a simple priority;

- All of the four approaches proposed are non-exhaustive, useful separately but not liable to be implemented and applied together;

The four constitutive approaches for an open and fair assessment of AI are enumerated as follows.

\subsection{Anthropomorphisation}

In this constitutive approach, the purposive construct is that artificial intelligence as a human artefact must be assumed as a technological entity that has certain substantive and operational attributes. In the hypothesis, the substantive and operative attributes of $\mathrm{AI}$ are limited of aesthetic nature and are immaterial, which means that these attributes are not directly bound by the technological features of AI as in the conceptions of technology sciences. These aesthetic attributes are equally recognized in all of the four approaches proposed in the paper and can be derived by a constitutionalist adjudication and interpretation of the characteristics of AI. The characteristics of the approach of anthropomorphisation are enumerated as follows: 
- The approach is premised on the basis that AI requires to be understood as a transformable legal entity, i.e., artificial intelligence possesses stimulating characteristics and can develop its own limited empathy;

- The empathy developed by AI is probabilistic by activities but is deterministic by observations because the same practicable empathy developed by AI is adaptive incoherent human environments. This is practicable in case of any technological asset developed. Therefore, by practice and purpose, AI is capable to develop adaptive empathy;

- The activities of AI are central to (a) the environment in which it resides and learns, and (b) the technological features of the AI that enable its learning and executability;

- The attributes of human entities are transformative and adaptive, by their psychological and biological means coupled with external and additional circumstances;

- Every substantive and operational aesthetic attribute of AI, therefore, must be separately adjudicated and interpreted without a pre-empted intent of correlation between the human and AI stimuli \& their practicable empathies;

- The adjudication of the aesthetic attributes of AI must uphold the concept of anthropomorphism, which means that the adjudication itself must recognize that AI as a technology is capable of mapping and resembling certain parameters of human characteristics;

The feature of the approach is that the relation between humans and technology is recognized in an intimate and more perfect method and that the relation between technology and human entities is not limited to the argument of (1) affirmative scientific humanism; and (2) material \& public welfare. The approach also enables by principle to widen the diversity of outcomes that can be sought through ethnographic analyses of the human-AI aesthetics that is observed.

\subsection{Naturalization}

The constitutive approach of naturalization signifies towards the appreciation and careful replenishment of the aesthetic attributes of AI beyond the initial recognition of the attributes. The approach focuses on the pragmatic scope of technology-led empathy by practice recognized by law and is subject to proper adjudication. The characteristics of the approach of naturalization are enumerated as follows:

- The aesthetic attributes of AI must be revised and improved for a conditioned and liberalized assessment of the human-AI aesthetics under observation;

- The learning and executability functions \& mechanisms of AI that are closely connected with the technological features of AI must be designed and based on (a) environment reconciliation, and (b) environment realization. Both methodologies must keep the individualistic and indigenous attributes of human society the first priority to assess the empathy of AI, and then resort towards industrial and governance goals as the secondary priority, wherein the second priority must not override the primary priority;

- The method of environment reconciliation means that the environment resided by AI must be learnt and normalized by AI to ensure proper and reasonable knowledge of the environment in which the AI can reconcile its own transformative features; 
- The method of environment realization follows the method of environment reconciliation, which means that the practical and exercisable empathy of AI in its general resident environment must be explainable, adaptive and self-assessable;

- The test of explanability, adaptability and self-assessment (hereinafter EAS test) in adjudicating the empathy of AI must, in principle, not be overridden by interpretable, perceptible and learning capabilities of AI in order to ensure a fair, just and reasonable assessment of the aesthetic attributes of AI;

- The EAS test for the purpose of adjudicating the empathy of AI, in principle, must prove that the method of environment reconciliation and realization developed within AI must ensure that the entrepreneurial ethics and design (hereinafter EED) behind and in observation (irrespective of positive or zero correlation) must allow a liberalized and freer development of the human data subjects, and such EED must not cause, contribute or render any undue influence over the obscure and delicate privacies pre-emptively reserved by human data subjects;

The feature of this approach is that it helps in a deeper assessment of the aesthetic attributes of the AI in alignment (no correlation) with the technological features of AI, making vicarious and entitative liabilities of the AI deterministic through a test that is based on the empathy of AI by practice and purpose. It also protects the fair, objective and democratized mandate of entrepreneurial ethics and design to carefully assess the anomalies of technology ethics and practices of both political and apolitical nature.

\subsection{Techno-Socialization}

The constitutive approach of techno-socialization is based on the position that artificial intelligence as a human artefact must reside in a socialized, regularized and improved human real-time environment. The approach of techno-socialization aims at proper scrutiny and understanding of the delicacy, sensitivity and scope of the human environment. Further, the human environment implied includes both real and material environments \& the cyberspace in which the AI resides. The characteristics of the approach are enumerated as follows:

- The social environment of the data subjects must be properly scrutinized and assessed. The nature of assessment and scrutiny must not involve an absolutist and restrictive approach based on legal formalism by practice and in principle;

- The technological rubric of cyberspace and material environments must be equitably and coherently surveyed and estimated;

- The ethical autonomy of data subjects must be safeguarded and kept as an overriding priority against the aesthetic liberties of AI, provided that the overriding effect must exist in principle and not achieved by design and practice by the industrial and governing entities responsible and tested for limited liability;

- The real-time environment must be designed and preserved taking into consideration the economic and social design of the environments in principle and practice;

- The environment must be sociable, regularized and replenishable itself for the AI to enable the self-transformation of the aesthetics of AI; 
- A sociable environment means the empathy of AI must be distinctively explainable, adaptive and self-assessable, and this distinctiveness must not be influenced or damaged;

- A regularized environment means that the social and economic rubric of the environment must be regularized through ethical and fair practices;

- A replenishable environment means that the inherent anthropomorphic liberties of humans must seek ethically autonomous and libertarian change by practice;

The feature of the approach is that the characteristics of a human environment are now required to be assessed and resolved adequately for a freer and ethical AI. The approach also endorses the libertarian model of fundamental rights and liberties \& affirms that human liberty and privacy is absolute and integral to industrial and governance responsibilities and liabilities.

\subsection{Enculturation}

The constitutive approach of enculturation involves an ethnographic assessment and liberalization of the identities of human data subjects and the cultivation of cultural identities. The purpose of enculturation is an essential rapprochement of cultures and identities \& the preservation of the cultural \& identity rights and liberties of human entities in the aesthetics involving the human entities and AI. The characteristics of the approach are enumerated as follows:

- The protection of cultural heritage and identities directly and indirectly associable must be encouraged, harmonized and normalized;

- The safeguard of cultural and identity liberties must be an intermediary liability on the entity that breaches the safeguard;

- The data involving identities (including culture) and their heritage must be treated as identity-oriented footprints in case of AI, which means that every such data will be coherent and connected and is useful in assessing the empathy, learning and executability of the AI;

- The probabilistic mechanisms of AI under deterministic assessments are not deprived of creative aesthetic liberties to exercise enculturation;

- The protection of cultures must not be defined by an absolutist, rudimentary and strict adjudication and interpretation involving the issues related to the same. Instead, the protection of cultures must be put into practice by naturalized, real, communitycentred, non-isolated, ethically autonomous means of adjudication;

- The obscurity of identity-oriented footprints (based on assessment, record or question of fact) must be recognized in assessing the attributes of AI;

- The identity-oriented footprints can be generated, learnt \& manifestly determined by AI within its self and vicarious liabilities;

The feature of this approach is that the semblance of human culture and empathy $\&$ technology ethics is determined, recognized and put into the principle to ensure that the role of technology as a dynamic force to drive human cultures is automated and regularized. 


\section{Conclusions}

The conclusions of the paper rest on the implications of the key challenges as analyzed in the previous sections and the characteristics of the constitutive approaches put in the hypothesis. It is proposed that the approaches and challenges asserted in the paper are reflective in nature. It is proposed that AI is a special class of technology, and its different and disruptive behaviour in practice and observation is a preemptive basis of the constitutive approaches and the challenges enumerated in the paper:

- There is a lack of consensus over automating ethnographic virtues of AI by practice and purpose in nations. The approaches of conventional and constitutive morality assumed in the legal ethics of data protection regime adopted by countries have conflicts at a global level to some extent because of the ethnocentric nature of the AI Ethics principles endorsed in the West;

- The stimulating structure of AI is data-centric, probabilistic and absolutist, which can improve and change its normal definitiveness with better scientific advancements in the technology involved;

- The resemblance of AI in tech-oriented welfare cannot be stretched to its utilitarian outset: for public and social welfare, the design of AI should be human-centric, naturalist and entitative;

- A generational ecosystem of technology like AI must lead with better tech-led socialization. There should be a society-observant renovation of AI systems to encourage proper skill development and diversification of employment opportunities;

- Companies that develop and use AI may certainly lack popular or general confidence of the public as the aesthetics involving the development of AI under the market economy model lacks human value, originality and replenishment, and instead causes more technology distancing when it comes to a balance between the human capital and the AI capital required in industrial, innovation and government sectors;

- Data-truistic digital colonization makes the aesthetic relationship of AI and human entities complex and diverging, due to lack of autonomous empathy that is encultured and learnt by the AI;

- Utilitarian and industry-centric methods with isolation to individual quality assessment and development in business ethics do not convene the naturalistic and individual-centric capabilities of individuals and make AI vulnerable to technology distancing $[19,20]$. Thus, true accountability and real trust with regards AI can only be developed when indigenous and individual interests are appropriately considered;

The conclusions are therefore provided as follows:

- In a post-liberal international order, the ethos of AI can be protected and diversified by cutting the monopolistic nature of the utilitarian and market-centric approach of AI;

- The practices of entrepreneurial ethics and design among governments and companies must keep individual, indigenous and libertarian interests by practice as the key priority to avoid malpractices with respect to technology and its economics and social influence; 
- Social and economic nationalism under the politics of populism and redemption cannot be isolated when it comes to reforming the anomalies of globalization. The ethos of technological globalism must be protected, reconciled and improved to encourage a trust-based identity-affirmative order to cater and maintain the operant benefits of the rule-based international order;

- Technology diplomacy will require prevention of the damaging implications of the politics and practice of social, political and economic redemption \& protectionism among developing and developed economies instead of mere containment of the problems;

- The stature of AI must be considered entitative and self-transformative as a juristic entity through a dissected and federalized assessment as proposed in the constitutive approaches;

- The rule-based international order must not be led and dominated by ethnocentric foreign policy reservations in terms of developed and developing economies, when it comes to the democratization of technology and the life of the internet, because political and constitutional confidence is essential from the local level to the diplomatic level;

\section{References}

1. BAAI: Beijing AI Principles, 28 May 2019. https://www.baai.ac.cn/blog/beijing-ai-principles

2. Google Spain v AEPD and Mario Costeja González (2014)

3. OHCHR: Guiding Principles on Business and Human Rights (2011). https://www.ohchr.org/ Documents/Publications/GuidingPrinciplesBusinessHR_EN.pdf

4. Koskenniemi, M.: From Apology to Utopia: The Structure of International Legal Argument. Cambridge University Press, Cambridge (2005)

5. Tripković, B.: The metaethics of constitutional adjudication. Department of Law, European University Institute, Florence (2015)

6. Commission Nationale de l'Informatique et des Libertés (CNIL), France, European Data Protection Supervisor (EDPS), European Union, Garante per la protezione dei dati personali, Italy. Declaration on Ethics and Data Protection in Artificial Intelligence, 23 October 2018. https:// edps.europa.eu/sites/edp/files/publication/icdppc-40th_ai-declaration_adopted_en_0.pdf

7. NITI Aayog, Government of India: National Strategy for AI - Discussion Paper, June 2018. https://niti.gov.in/writereaddata/files/document_publication/NationalStrategy-for-AIDiscussion-Paper.pdf. Accessed 2 Jan 2020

8. European Union: Regulation (EU) 2016/679 of the European Parliament and of the Council of 27 April 2016 on the protection of natural persons with regard to the processing of personal data and on the free movement of such data, and repealing Directive 95/46/EC (2016)

9. VDW: Policy Paper on the Asilomar Principles on Artificial Intelligence, Asilomar (2017)

10. Madiega, T.: EU guidelines on ethics in artificial intelligence: context and implementation (PE 640.163), September 2019. https://www.europarl.europa.eu/RegData/etudes/BRIE/2019/640 163/EPRS_BRI(2019)640163_EN.pdf. Accessed 2 Jan 2020

11. European Commission: Ethics guidelines for trustworthy AI. European Commission, 8 April 2019. https://ec.europa.eu/digital-single-market/en/news/ethics-guidelines-trustwort hy-ai. Accessed 30 Dec 2019

12. Salmon, J.: The legacy of Jean Bodin: absolutism, populism or constitutionalism? Hist. Polit. Thought 17(4), 500-522 (1996) 
13. House of Representatives: Impeachment of Donald John Trump, President of the United States, 16 December 2019. https://docs.house.gov/billsthisweek/20191216/CRPT-116hrp t346.pdf

14. Koh, H.H.: The Trump Administration and International Law (2017). https://digitalcommons. law.yale.edu/fss_papers/5213

15. Dutta, S.: Determinants of Ethnocentric Attitudes in the United States (2009). https://paa 2009.princeton.edu/abstracts/91531

16. Grewal, J., Rakesh, V., Chattopadhyay, S., Hickock, E.: Report on Understanding Aadhaar and its New Challenges, 31 August 2016. https://cis-india.org/internet-governance/blog/rep ort-on-understanding-aadhaar-and-its-new-challenges

17. Akula, R., Liu, C., Saba-Sadiya, S., Lu, H., Todorovic, S., Chai, J.Y., Zhu, S.-C.: X-ToM: Explaining with Theory-of-Mind for Gaining Justified Human Trust, 15 September 2019. https://arxiv.org/abs/1909.06907

18. Pacey: Meaning of Technology. The MIT Press, Cambridge (1999)

19. Molla, R.: "Knowledge workers" could be the most impacted by future automation, 20 November 2019. https://www.vox.com/recode/2019/11/20/20964487/white-collar-automation-riskstanford-brookings

20. Manyika, J., Chui, M., Miremadi, M., Bughin, J., George, K., Wilmott, P., Dewhurst, M.: Mc Kinsey Global Institute: A Future That Works: Automation, Employment, and Productivity (2017). https://www.mckinsey.com/ /media/McKinsey/Featured\%20Insights/Digital\%20D isruption/Harnessing $\% 20$ automation $\% 20$ for $\% 20 a \% 20$ future $\% 20$ that $\% 20$ works/MGI-A-fut ure-that-works_Full-report.ashx 